\title{
Síndrome metabólico. Bases clínicas y fisiopatológicas para un enfoque terapéutico racional
}

\author{
Gonzalo Martínez $\mathbf{R}^{1, a}$, Rodrigo Alonso $K^{2}$, \\ Victoria N ovik $A^{3,4}$.
}

\section{Metabolic Syndrome. Clinical and pathophysiological basis for a rational therapeutical approach}

Regardless of the diagnostic criteria, the metabolic syndrome is found at least in $20 \%$ of the population. The adipose tissue plays an important role in the insulin resistance found in this syndrome. Free fatty acids released by intra-abdominal adipocytes produce an inflammatory and pro-thrombotic response and the persistance of the insulin resistance state, phenomenon termed lipotoxicity. This altered phenotype explains the development of the different components of the metabolic syndrome, such as hypertension, dyslipidemia and altered glucose metabolism. The treatment is based on weight loss and healthy lifestyle. A balanced diet, physical activity and avoidance of smoking are key management features. The use of drugs with pleiotropic effects, that inhibit the renin angiotensin aldosterone axis or acts on the peroxisome proliferator-activated receptors (PPAR) seems promising (Rev Méd Chile 2009; 137: 685-94).

(Key words: Insulin resistance; Metabolic Syndrome X; Obesity)

Recibido el 16 de noviembre, 2007. Aceptado el 28 de julio, 2008.

${ }^{1}$ Cátedra de Fisiopatología, Universidad de Valparaíso, Valparaíso, Chile. ${ }^{2}$ Unidad de Lípidos, Fundación Jiménez-Díaz, Madrid, España. ${ }^{3}$ Departamento de Endocrinología, Hospital Dr. Gustavo Fricke, Viña del Mar, Chile. ${ }^{4}$ Cátedra de Medicina Interna, Universidad de Valparaíso, Valparaíso, Chile.

aResidente de Medicina Interna, Universidad de Valparaíso, Valparaíso, Chile.

$\mathrm{E}^{1}$ síndrome metabólico (SM) corresponde a la asociación de una serie de anormalidades metabólicas que determinan un mayor riesgo de padecer enfermedad cardiovascular y diabetes mellitus (DM) en el individuo afecto ${ }^{1-4}$.

Obesidad central, hipertensión arterial, dislipidemia y alteración del metabolismo de los hidra-

Correspondencia a: Victoria Novik A. Asturias 1750, casa 6, Viña del Mar Alto. Fono: 32-2485686.

E mail: victorianovik@gmail.com tos de carbono son condiciones que se asocian frecuentemente, por lo cual se ha buscado un sustrato que explique su relación. Si bien existe amplia controversia respecto a la real existencia del SM como entidad patológica y a la utilidad de un enfoque terapéutico particular ${ }^{2}$, recientes avances en el conocimiento de este síndrome permiten plantear una patogenia común, reconociendo a la obesidad abdominal como una condición fundamental en su desarrollo, por medio de una serie de mecanismos que interrelacionan sus distintos componentes ${ }^{5}$. 
En la presente revisión se discutirán el rol del SM como factor de riesgo cardiovascular (FRCV) y la importancia de la obesidad central en su patogenia, estableciendo un fundamento lógico y racional sobre el cual basar las diferentes intervenciones terapéuticas en los pacientes con SM.

\section{DEFINICIÓN Y PREVALENCIA}

La definición del SM varía según los distintos consensos internacionales. Cuatro grupos han planteado criterios diagnósticos: la Organización Mundial de la Salud (OMS), el Grupo de Estudio para la Resistencia a la Insulina (EGIR), el consenso del National Cholesterol Education Program Adult Treatment Panel III (NCEP ATP III) y el consenso de la Federación Internacional de Diabetes (IDF) ${ }^{4}$. En general, mantienen los criterios, pero discrepan en su medición y puntos de corte. Los consensos de la IDF y del NCEP ATP III son los más utilizados y difundidos. Este último requiere la presencia de al menos 3 de los siguientes 5 criterios para el diagnóstico: Obesidad central, elevación de triglicéridos, C-HDL bajo, hipertensión arterial y alteración de la glicemia de ayunas (mayor a $110 \mathrm{mg} / \mathrm{dl}$ ), sin establecer categorías entre los factores ${ }^{3}$. Posteriormente, la American Heart Association/National Heart, Lung and Blood Institute (AHA/NHLBI) sugirió considerar $100 \mathrm{mg} /$ dl como punto de corte para la glicemia de ayunas ${ }^{1}$. La Federación Internacional de Diabetes (IDF) establece como requisito básico la presencia de obesidad central y la medición de la circunferencia abdominal ${ }^{6}$ (Tabla 1 ).

Dependiendo del criterio empleado, la edad, el género y la raza, la prevalencia del SM varía marcadamente. Así, aumenta con la edad independientemente del criterio utilizado, y es más alta en varones cuando se utilizan los criterios de la OMS y del EGIR. Con los criterios de la OMS, la prevalencia para varones y mujeres menores de 55 años es de $14 \%$ y $4 \%$, respectivamente, y de $31 \%$ y $20 \%$ en los mayores de esa edad 7 . En Estados Unidos de Norteamérica, utilizando los criterios NCEP ATP III, la prevalencia general es $24 \%$, y aumenta directamente con la edad y el índice de masa corporal. En jóvenes norteamericanos entre 12 y 19 años la prevalencia es $4,2 \%$ y supera $40 \%$ en la población de 65 a 69 años ${ }^{8}$.
En la Encuesta Nacional de Salud, realizada en Chile el año 2003, 22,6\% de la población adulta cumplía los criterios diagnósticos del NCEP ATP III, con cifras similares para hombres y mujeres. Al igual que en el estudio NHANES III, se observó un aumento de la prevalencia a mayor edad de la población estudiada 9 .

\section{EL SÍNDROME METABÓLICO COMO FACTOR DE RIESGO CARDIOVASCULAR}

Se ha demostrado la asociación del SM con un mayor riesgo de desarrollar enfermedad cardiovascular. El estudio DECODE, en pacientes con SM no diabéticos, mostró riesgos ajustados mayores de enfermedad cardiovascular (OR 2,26 para hombres y 2,78 para mujeres) y de muerte (OR $1,44$ para hombres y 1,38 para mujeres $)^{10}$. En el estudio INTERHEART, distintos componentes del $\mathrm{SM}$, como dislipidemia aterogénica, hipertensión arterial, DM y obesidad abdominal fueron reconocidos como factores de riesgo cardiovascular (FRCV) independientes a nivel poblacional, y su asociación determinó un riesgo mucho mayor ${ }^{11}$.

En un metaanálisis reciente, con 172.573 pacientes, se concluyó que hay un riesgo cardiovascular y de muerte significativamente mayor en personas con SM y que éste no es solamente explicable por sus componentes por separado. El mayor problema de este metaanálisis es la heterogeneidad en las definiciones de SM de los trabajos incluidos ${ }^{12}$.

La Asociación Americana de Diabetes reconoce que algunos FRCV tienden a asociarse, pero plantea la falta de evidencia respecto a si en el SM "el total es más que la suma de las partes"13. Gerald Reaven ha planteado dudas acerca de la utilidad y la capacidad predictiva del diagnóstico de SM. Considera la medida de la circunferencia abdominal inadecuada para evaluar grasa visceral y cuestiona la utilidad de hacer el diagnóstico de $\mathrm{SM}$, ya que cree que no tiene implicancias terapéuticas $^{14-16}$.

Independientemente de la utilidad del diagnóstico del SM, un enfoque global del paciente, considerando a la obesidad como un factor esencial y orientado a intervenciones sistémicas que logren identificar y reducir en conjunto estos FRCV, representa un acercamiento racional y 
Tabla 1. Criterios diagnósticos para síndrome metabólico

\begin{tabular}{|c|c|c|c|c|}
\hline $\begin{array}{l}\text { Medida } \\
\text { clínica }\end{array}$ & OMS & EGIR & NCEP ATP III & IDF \\
\hline $\begin{array}{l}\text { Resistencia a } \\
\text { la insulina }\end{array}$ & $\begin{array}{l}\text { DMT2, GAA, IGO o } \\
\text { disminución de } \\
\text { sensibilidad a la } \\
\text { insulina más } 2 \text { de los } \\
\text { siguientes }\end{array}$ & $\begin{array}{l}\text { Insulina } \\
\text { plasmática >p75 } \\
\text { más dos de los } \\
\text { siguientes }\end{array}$ & $\begin{array}{l}\text { Ninguno, pero al } \\
\text { menos } 3 \text { de los } 5 \\
\text { siguientes }\end{array}$ & Ninguno \\
\hline $\begin{array}{l}\text { Obesidad } \\
\text { central }\end{array}$ & $\begin{array}{l}\text { Razón cintura/cadera } \\
\text { Hombres: }>0,9 \\
\text { Mujeres: }>0,85 \\
\text { o IMC }>30\end{array}$ & $\begin{array}{l}\mathrm{CA} \geq 94 \mathrm{~cm} \text { en } \\
\text { hombres } \\
\geq 80 \mathrm{~cm} \text { en } \\
\text { mujeres }\end{array}$ & $\begin{array}{l}\mathrm{CA} \geq 102 \mathrm{~cm} \text { en } \\
\text { hombres } \\
\geq 88 \mathrm{~cm} \text { en mujeres }\end{array}$ & $\begin{array}{l}C A \geq 90 \mathrm{~cm} \text { en } \\
\text { hombres } \\
\geq 80 \mathrm{~cm} \text { en mujeres } \\
\text { más } 2 \text { de los siguientes } \\
\text { criterios }\end{array}$ \\
\hline $\begin{array}{l}\text { Lípidos } \\
\text { (mg/dl) }\end{array}$ & $\begin{array}{l}\mathrm{TG} \geq 150 \text { o } \\
\mathrm{C}-\mathrm{HDL}<35 \text { en } \\
\text { hombres } 0<39 \text { en } \\
\text { mujeres }\end{array}$ & $\begin{array}{l}\text { TG } \geq 150 \text { o } \\
\text { C-HDL }<39 \text { en } \\
\text { hombres y } \\
\text { mujeres }\end{array}$ & $\begin{array}{l}\mathrm{TG} \geq 150 \text { o } \\
\mathrm{C} \text {-HDL }<40 \text { en } \\
\text { hombres } 0<50 \text { en } \\
\text { mujeres }\end{array}$ & $\begin{array}{l}\mathrm{TG} \geq 150 \text { o } \\
\mathrm{C}-\mathrm{HDL}<40 \text { en } \\
\text { hombres } 0 \\
<50 \text { en mujeres }\end{array}$ \\
\hline $\begin{array}{l}\text { Presión arterial } \\
(\mathrm{mmHg})\end{array}$ & $\geq 140 / 90$ & $\geq 140 / 90$ & $\geq 130 / 85$ & $\geq 130 / 85$ \\
\hline Glucosa & DMT2, GAA, IGO & $\begin{array}{l}\text { GAA, IGO, pero } \\
\text { no DMT2 }\end{array}$ & $\begin{array}{l}>110 \mathrm{mg} / \mathrm{dl} \\
\text { (incluida DMT2)* }\end{array}$ & $\begin{array}{l}\text { Glicemia en ayunas } \\
>100 \mathrm{mg} / \mathrm{dl} \text { o DMT2 } \\
\text { previamente diagnosticada }\end{array}$ \\
\hline
\end{tabular}

DMT2 indica diabetes mellitus tipo 2; GAA, glicemia de ayuno alterada; IGO, intolerancia a la glucosa oral; IMC, índice de masa corporal; TG, triglicéridos; $\mathrm{CA}$, circunferencia abdominal

*Modificado posteriormente por AHA/NHLBI a $>100 \mathrm{mg} / \mathrm{dl}$

Nomenclatura clasificaciones:

OMS: Organización Mundial de la Salud

EGIR: Grupo Europeo para el Estudio de la Resistencia a la Insulina

ATP III: National Cholesterol Education Program Adult Treatment Panel III

IDF: Federación Internacional de Diabetes

Adaptado de las referencias 1 y 6 .

necesario para alcanzar el objetivo de una prevención cardiovascular adecuada.

\section{SíNDROME METABÓLCO Y DIABETES MELUTUS}

La existencia de SM y DM como entidades independientes es motivo de constante controversia, cuyo análisis sobrepasa los objetivos de este artículo ${ }^{17}$. Cabe mencionar que las discrepancias son motivadas en parte por la heterogeneidad de los criterios diagnósticos de SM, ya que unos excluyen a la DM como requisito para el diagnóstico, mientras otros sólo requieren la existencia de glicemia de ayuno alterada, sin especificar un valor superior (con la consiguiente inclusión de pacientes diabéticos) (Tabla 1). A su vez, en pacientes que cumplen los criterios de $\mathrm{SM}$, pero que no tienen $\mathrm{DM}$, el riesgo de desarrollar esta enfermedad está aumentando alrededor de 6 veces $^{18}$. Por otra parte, en un análisis post-hoc del Verona Diabetes Complications Study, la presencia de SM en individuos diabéticos fue un FRCV independiente, al aumentar en cinco veces los eventos cardiovasculares ${ }^{19}$. 


\section{Fisiopatología}

Uno de los sustentos de la existencia del SM como entidad clínica es una patogenia común para la mayoría de los casos, si bien esto no constituye una condición sine qua non para el diagnóstico de $\mathrm{SM}^{4}$.

Inicialmente se consideró al SM como la expresión fenotípica de la resistencia a la insulina (RI). Ésta corresponde a una respuesta subnormal del organismo a la acción insulínica en los tejidos periféricos. Secundario a la RI, las células $\beta$ pancreáticas aumentan su secreción de insulina compensatoriamente, produciendo hiperinsulinemia, la cual se pensó era responsable de muchos de los fenómenos encontrados en el SM ${ }^{20,21}$.

Actualmente, la visión fisiopatológica del SM ha cambiado, racionalizando y justificando nuevas intervenciones terapéuticas. Los principales conceptos en la patogenia del SM son:

A. Considerar a la obesidad como el eje central patogénico del SM y a la RI como una consecuencia de ésta. B. Reconocer al adipocito como una célula inflamatoria y directamente contribuyente a la génesis del SM. C. Considerar los efectos biológicos no clásicos de la insulina, que explican muchas de las alteraciones observadas en el SM.

A. Obesidad y síndrome metabólico. El tejido adiposo en los obesos es insulinorresistente, lo que eleva los ácidos grasos libres (AGL) en el plasma. Éstos tienen un efecto directo en los órganos diana de la insulina, como hígado y músculo, mediante acciones específicas que bloquean la señalización intracelular del receptor de insulina ${ }^{22}$. Este fenómeno, conocido como lipotoxicidad, sería responsable de la RI en estos órganos y la falta de regulación pancreática a la glicemia elevada ${ }^{23,24}$. Además, los AGL serían capaces de aumentar el estrés oxidativo, el ambiente proinflamatorio sistémico y disminuir la reactividad vascular ${ }^{24}$. Los AGL, a través de la inhibición de la acción insulínica, determinan una supresión insuficiente de la lipasa hormonosensible del adipocito, mayor incremento de AGL y autoperpetuación del ciclo.

En los pacientes con SM el tejido adiposo es de predominio central, asociado a mayor cantidad de grasa visceral comparado con la distribución periférica de ésta ${ }^{25}$. Los adipocitos de la grasa visceral son metabólicamente más activos, liberando mayor cantidad de $\mathrm{AGL}^{26}$ y citoquinas inflamatorias $^{27}$ que drenan directamente al hígado a través de la circulación portal.

B. El adipocito como célula inflamatoria. El estado proinflamatorio asociado a la obesidad (y por tanto al SM) se explica por la presencia de células inflamatorias entre las células adipocitarias y por la actividad inflamatoria propia de los adipocitos. Se ha observado un aumento de moléculas como el factor de necrosis tumoral $\alpha$ (FNT- $\alpha)$, inhibidor del activador del plasminógeno 1 (PAI-1), interleuquina 6 y leptina y disminución de la adiponectina ${ }^{28}$, las cuales tienen la capacidad de modular reacciones inflamatorias, trombóticas y vasoactivas.

$\mathrm{El}$ efecto de las citoquinas inflamatorias sobre la sensibilidad insulínica es conocido. El FNT- $\alpha$ produce una fosforilación anormal del sustrato del receptor de la insulina (IRS), que a su vez produce una nueva fosforilación en un sitio incorrecto del receptor de insulina (serina en lugar de tirosina) y con esto una alteración en su transducción ${ }^{29}$. El mecanismo por el cual las citoquinas inflamatorias alteran la fosforilación normal del receptor de insulina es mediante la activación de "Suppressor of cytokine signalling 3" (SOCS-3), una proteína capaz de interferir con dicha fosforilación y también degradar al IRS ${ }^{30}$.

La adiponectina es una citoquina antiinflamatoria producida exclusivamente por los adipocitos. Es capaz de aumentar la sensibilidad a la insulina e inhibir varios pasos en el proceso inflamatorio. También disminuiría la producción hepática de glucosa y la lipólisis. La disminución de adiponectina se asocia, en modelos experimentales y clínicos, con SM y progresión de enfermedad cardiovascular ${ }^{31}$.

Los alimentos también se han involucrado con el estado proinflamatorio asociado a la obesidad. Aljada y cols $^{32}$ mostraron que la ingesta de un menú de comida rápida era capaz de producir un estado proinflamatorio a través de la activación del factor nuclear kappa B (FN-кB). Esto mismo se ha repetido al realizar una infusión intravenosa de triglicéridos en sujetos sanos ${ }^{28}$. Desde este punto de vista, parece razonable plantear que una disminución en la ingesta calórica podría disminuir el estado proinflamatorio y de estrés oxidativo ${ }^{33}$ (Figura 1). 


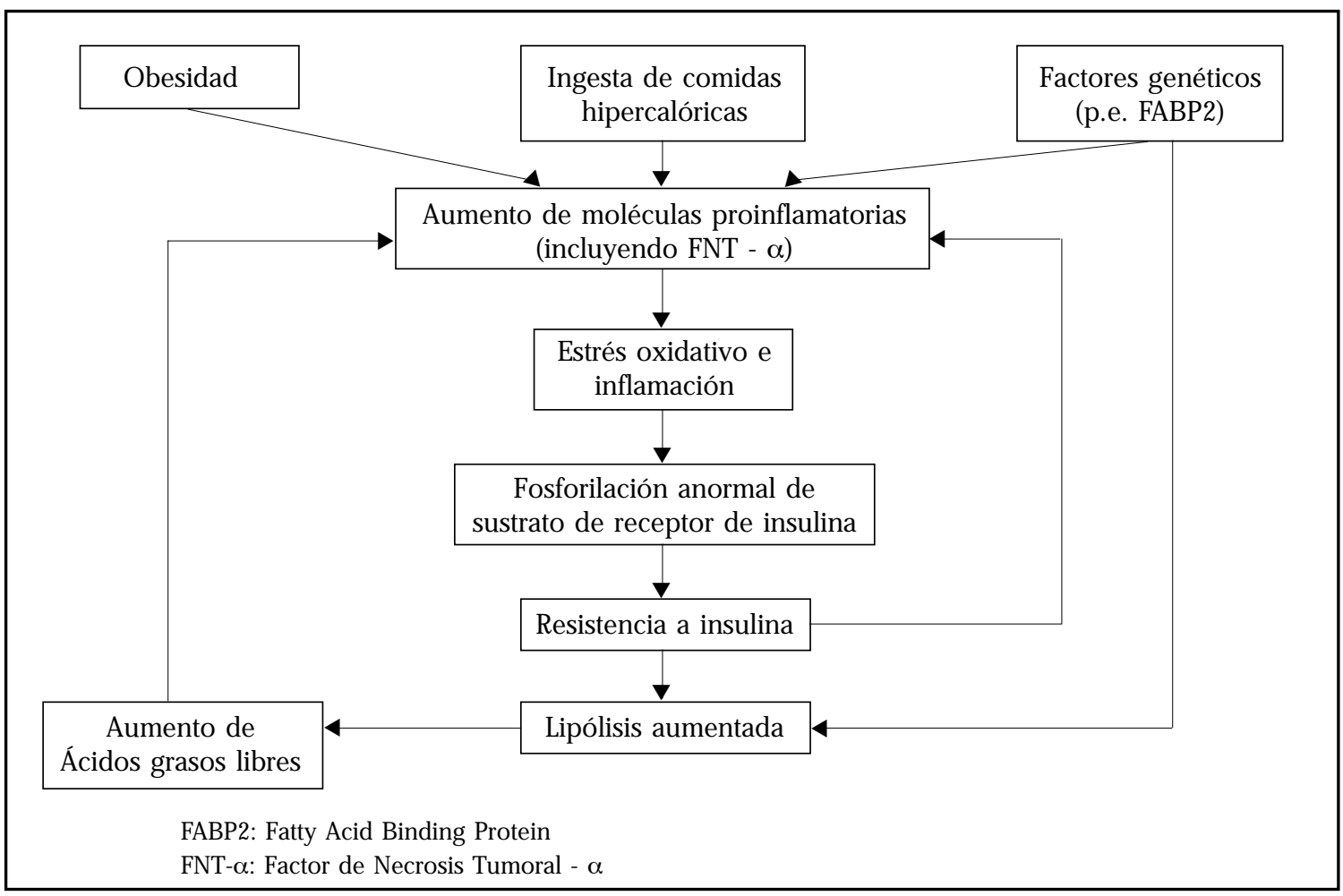

Figura 1. Hipótesis inflamatoria del Síndrome Metabólico. Adaptado de la referencia 22.

C. Efectos no clásicos de la insulina. La insulina posee efectos vasodilatadores, antitrombóticos, antiinflamatorios, antioxidantes y natriuréticos, los que se verían alterados en caso de $\mathrm{R}^{22}$. Estos efectos explican algunas de las alteraciones observadas en el $\mathrm{SM}$, especialmente en cuanto al mayor riesgo aterotrombótico. Así, la RI se transforma en un estado capaz de determinar un mayor riesgo de eventos cardiovasculares per se y no sólo asociado a los FRCV independientes. Las consecuencias sistémicas de la RI determinan un aumento de las especies reactivas de oxígeno y disminución de la actividad de la óxido nítrico sintetasa endotelial (eNOS). Esto se acompaña de aumentos a nivel local y sistémico en endotelina y angiotensina, potentes moléculas vasoconstrictoras e inflamatorias, que también están involucradas en la generación de estrés oxidativo ${ }^{34}$. Lo anterior conduce a un estado inflamatorio crónico en la pared vascular y a disfunción endotelial, que finalmente determinará la ateromatosis y el eventual episodio agudo de la placa ${ }^{35}$.
D. Factores genéticos. Explicarían la RI en individuos no obesos, poblaciones asiáticas y parientes directos de personas diabéticas. El polimorfismo de la "Fatty Acid Binding Protein 2" (FABP2) es uno de los genes candidatos que han sido asociados con RI y obesidad ${ }^{36}$. Esta proteína participa en el transporte de ácidos grasos de cadena larga a nivel intestinal. Los portadores de un alelo específico (Thr54) en FABP2 tienen el doble de afinidad por ácidos grasos de cadena larga que aquellos con la forma nativa, lo cual podría llevar a un aumento de los AGL en la circulación con la consecuente lipotoxicidad.

E. Rol de los PPAR (Receptor del proliferador peroxosimal activado). Los PPAR son receptores intracelulares capaces de modular el funcionamiento de los órganos metabólicamente activos. Existen 3 tipos de PPAR $(\alpha, \beta$ y $\gamma)$. Los PPAR $\gamma$ son esenciales para la diferenciación y proliferación normal de los adipocitos, como también para el 
almacenamiento de los ácidos grasos en ellos. Son capaces de aumentar la síntesis de adiponectina y de evitar la salida de AGL a la circulación ${ }^{37}$. No está clara la regulación endógena de los PPAR $\gamma$, pero su modulación mediante agonistas puede determinar una disminución de la RI y, con esto, una mejoría en el SM.

\section{ENFOQUE TERAPÉUTICO}

El objetivo principal del tratamiento de individuos con SM es prevenir el desarrollo de enfermedad cardiovascular ${ }^{1}$. La terapia de primera línea está dirigida hacia la pérdida de peso y el control de los factores de riesgo mayores: C-LDL, hipertensión arterial y alteración en el metabolismo de hidratos de carbono. Por lo tanto, la evaluación inicial de todo paciente con SM debe considerar la valoración de su riesgo cardiovascular global.

La diferencia en el enfoque del manejo del SM como entidad clínica radica en optimizar el control integral de los factores de riesgo asociados mediante cambios en el estilo de vida, realización de actividad física, ingesta de una dieta equilibrada y suspensión del tabaquismo. La terapia farmacológica está indicada para los casos en que estas intervenciones no logren conseguir los objetivos planteados para los distintos factores de riesgo. No hay guías de manejo especiales para la hipertensión arterial, diabetes o dislipidemia en los pacientes con SM, por lo que debieran seguirse las guías o recomendaciones de práctica clínica vigentes ${ }^{38}$ (Tabla 2).

En la obesidad, el uso de fármacos está recomendado para pacientes seleccionados, en los cuales las modificaciones del estilo de vida no han tenido éxito. Existen tres drogas principales para el manejo de la obesidad. Orlistat inhibe la lipasa gástrica y pancreática, reduciendo en 30\% la absorción de grasas. Produce reducción de peso significativa respecto a placebo en el primer año ${ }^{39}$ y logra reducir significativamente algunos FRCV y la $\mathrm{R}^{40}$. Sibutramina actúa inhibiendo la recaptación central de serotonina y norepinefrina, produciendo una sensación de saciedad. Reduce el peso en 4,6\% comparado con placebo ${ }^{41}$, lo que se asocia a mejoría en los niveles de triglicéridos, C-HDL y glicemia en población obesa no diabética ${ }^{42,43}$. Rimonabant se había incorporado a este arsenal terapéutico. Actúa bloqueando al receptor canabinoide tipo 1. Comparado con placebo, redujo el peso en un promedio de $8,6 \mathrm{~kg}$ versus $2,3 \mathrm{~kg}^{44}$. Además de disminuir la ingesta alimentaria, inhibe la lipogénesis hepática y adipocitaria y aumenta las concentraciones de adiponectina ${ }^{45}$, mejorando el perfil lipídico y el control glicémico y disminuyendo el número de pacientes con $\mathrm{SM}^{46}$. Rimonabant ha sido recientemente retirado del mercado farmacéutico por riesgo de depresión severa

La cirugía bariátrica es usada cada vez más frecuentemente para el tratamiento de la obesidad severa, logrando una reducción de peso de $23,4 \%$ a los 2 años y 16,1\% a los 10 años de seguimiento, lo que se acompaña de una mejoría en los niveles de triglicéridos, C-HDL, glicemia, presión arterial y uricemia y una menor incidencia de diabetes ${ }^{47}$. La prevalencia de SM en obesos mórbidos sometidos a este tipo de cirugía se reduce hasta $95 \%$ al cabo de 1 año ${ }^{48}$.

Para el tratamiento de la dislipidemia, el grupo farmacológico de elección son las estatinas. Un análisis post-hoc del estudio STELLAR mostró el efecto beneficioso de todas las estatinas sobre los parámetros lipídicos en pacientes con $\mathrm{SM}^{49}$. Debido al alto riesgo cardiovascular de estos pacientes, se suele requerir la asociación con otros fármacos. Los fibratos pueden ser útiles, ya que por su efecto sobre PPAR- $\alpha$ aportan beneficios adicionales en los niveles de triglicéridos y C-HDL ${ }^{50,51}$.

Considerando la fisiopatología del SM, se ha postulado que la inhibición del sistema renina angiotensina aldosterona (RAA) podría tener efectos beneficiosos más allá de su efecto antihipertensivo ${ }^{34}$. El bloqueo del sistema RAA con ramipril puede prevenir la aparición de diabetes en individuos con alto riesgo cardiovascular ${ }^{52}$, pero no así en pacientes de bajo riesgo ${ }^{53}$. El estudio LFE mostró que losartán fue más efectivo que atenolol en reducir la mortalidad y morbilidad cardiovascular en pacientes diabéticos, hipertensos y con hipertrofia ventricular izquienda $^{54}$. Telmisartán, además de bloquear el receptor de angiotensina, es un agonista parcial de PPAR- $\gamma$, lo cual le permitiría una acción insulinosensibilizadora, con reducción de la glicemia plasmática y los niveles de insulina ${ }^{55}$.

En aquellos pacientes con metabolismo de los hidratos de carbono alterado, metformina parece ser el fármaco más adecuado ${ }^{56}$. Además de controlar la glicemia en un importante número de 
Tabla 2. 0 bjetivos y recomendaciones para el manejo del síndrome metabólico

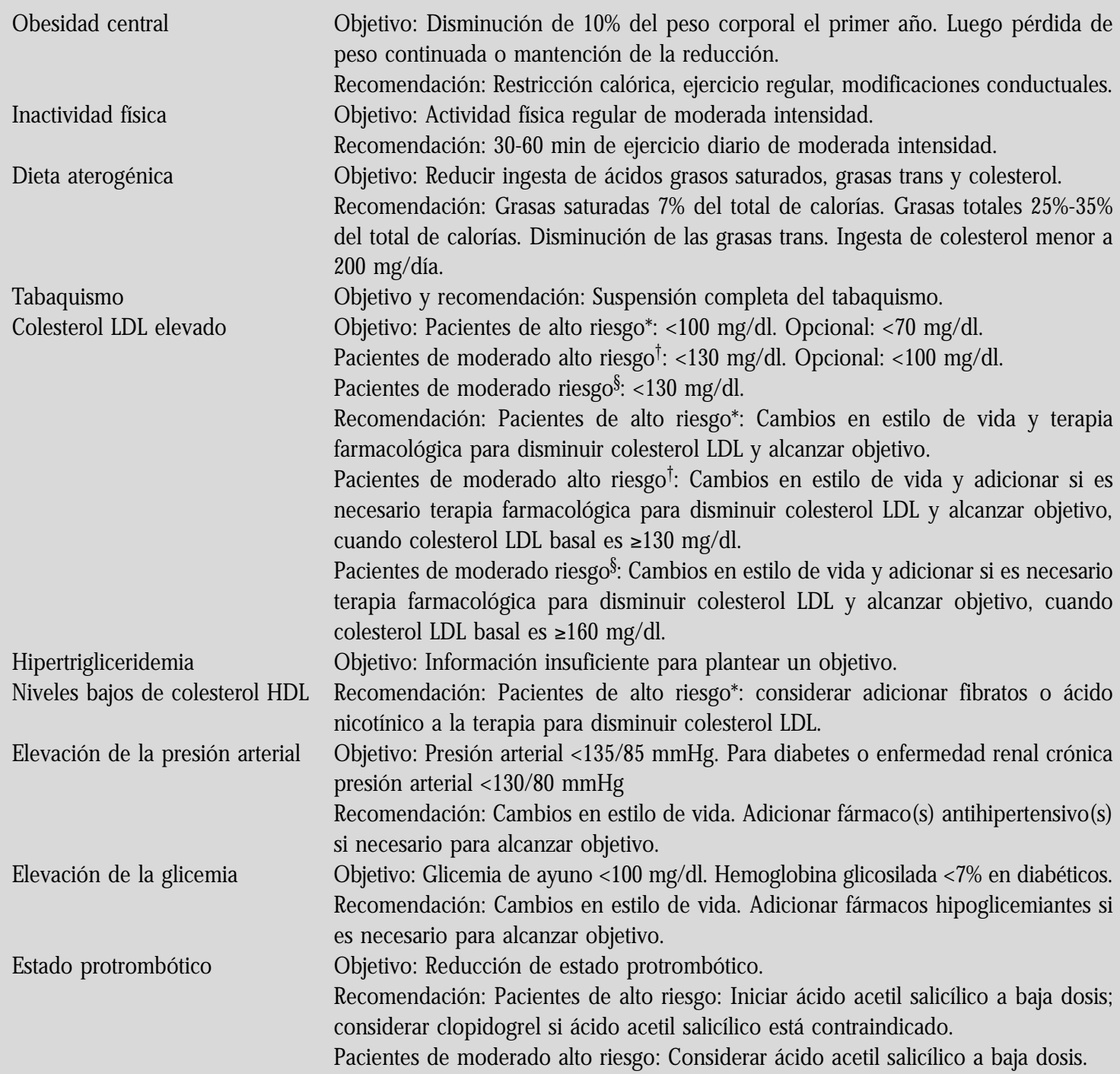

* Pacientes de alto riesgo. Aquellos con enfermedad cardiovascular ateroesclerótica establecida, diabetes mellitus o riesgo de enfermedad coronaria a 10 años $>20 \%$.

† Pacientes de moderado alto riesgo. Aquellos con riesgo de enfermedad coronaria a 10 años entre 10\%-20\%.

$\S$ Pacientes de moderado riesgo. Aquellos con síndrome metabólico pero riesgo de enfermedad coronaria a 10 años $<10 \%$. Adaptado de la referencia 4.

pacientes, mejora la reactividad vascular en pacientes con SM y glicemias normales ${ }^{57}$. En el estudio UKPDS, el grupo que recibió metformina tuvo una reducción de la mortalidad cardiovascu- lar y global al compararlo con los pacientes que recibieron sulfonilureas o insulina ${ }^{58}$. La asociación de metformina con pioglitazona ha mejorado el perfil lipídico en pacientes con $\mathrm{SM}^{59}$. Si bien el 
estímulo de PPAR- $\gamma$ por rosiglitazona pudiera ser beneficioso, no ha logrado reducir la mortalidad cardiovascular y actualmente su seguridad ha sido cuestionada ${ }^{60}$.

\section{CONCLUSIONES}

El SM se ha convertido en una pandemia, gatillado por la creciente prevalencia de obesidad y estilos de vida no saludables. Independientemente de los criterios diagnósticos que se utilicen, el SM afecta cerca del $20 \%$ de la población adulta.

El tejido adiposo parece ser el responsable del estado de resistencia a la insulina que caracteriza a este síndrome. La lipotoxicidad producida por los AGL liberados de los adipocitos intraabdominales determina una respuesta inflamatoria y pro-

\section{REFERENCIAS}

1. Grundy S, Cleeman J, Daniels S, Donato K, Eckel R, FRANKLIN B ET AL. Diagnosis and Management of the Metabolic Syndrome. An American Heart Association/National Heart, Lung and Blod Institute Scientific Statement. Circulation 2005; 112: 2735-52.

2. Friedewald V, Grundy S, Gotto A, Haffner S, Denke M, HoLANDER P, RoBerTs W. The Editor's Roundtable: The Metabolic Syndrome. Am J Cardiol 2007; 99: 382-9.

3. Executive Summary of The Thind Report of The National Cholesterol Education Program (NCEP) Expert Panel on Detection, Evaluation, And Treatment of High Blood Cholesterol In Adults (Adult Treatment Panel III). JAMA 2001; 285: 2486-97.

4. Eckel R, Grundy S, Zimmet P. The metabolic syndrome. Lancet 2005; 365: 1415-28.

5. Haslam D, James W. Obesity. Lancet 2005; 366: 1197209.

6. Alberti K, Zimmet P, Shaw J. Metabolic syndrome-a new world-wide definition. A Consensus Statement from the International Diabetes Federation. Diabet Med 2005; 23: 469-80.

7. Consenso del Grupo de trabajo Resistencia a ia Insulna, de La Sociedad Española de Diabetes. La resistencia a la insulina y su implicación en múltiples factores de riesgo asociados a diabetes mellitus tipo 2. Med Clin (Barc) 2002; 119: 458-63.

8. Ford ES, GiLES WH, DieTZ WH. Prevalence of the metabolic syndrome among US adults: findings from the third National Health and Nutrition Examination Survey. JAMA 2002; 287: 356-9. trombótica y la perpetuación del estado de insulino-resistencia. Este fenotipo alterado explica la aparición de los distintos componentes del SM, como hipertensión arterial, dislipidemia y alteración en el metabolismo glucídico.

El tratamiento del SM se basa en la reducción de peso y en la adquisición de un estilo de vida saludable. Una dieta equilibrada, actividad física y suspensión del tabaquismo son medidas fundamentales en el manejo de estos pacientes. En el estado actual del conocimiento, los componentes del SM deben ser tratados de forma individual, buscando alcanzar los objetivos planteados por las guías de manejo respectivas. El uso de moléculas con efectos pleiotrópicos, que inhiban el sistema RAA o actúen sobre PPAR parece promisorio y orientan al estudio de nuevas terapias para estos pacientes.

9. Encuesta Nacional de Salud, Chile 2003, en http: // ep i.minsal.cl/epi/ html/invest/ENS/ InformeFinalENS.pdf.

10. Hu G, Qiao Q, Tuomilehto J, Balkau B, Borch-Johnsen $\mathrm{K}$, Pyorala K. Prevalence of the metabolic syndrome and its relation to all-cause and cardiovascular mortality in nondiabetic European men and women. Arch Intern Med 2004; 164: 1066-76.

11. Yussuf S, Hawken S, Ounpuu S, Dans T, Avezum A, LANAS F. Effect of potentially modifiable risk factors associated with myocardial infarction in 52 countries (the INTERHEART study): case-control study. Lancet 2004; 364: 937-52.

12. Gami A, Witt B, Howard D, Erwin P, Gami L, Somers V Y cols. Metabolic Syndrome and Risk of Incident Cardiovascular Events and Death. A Systematic Review and Meta-Analysis of Longitudinal Studies. J Am Coll Cardiol 2007; 49: 403-14.

13. Kahn R, Buse J, FerRannini E, Stern M. The Metabolic Syndrome: Time for a Critical Appraisal. Diabetes Care 2005; 28: 2289-304.

14. REAVEN G. The metabolic syndrome: is this diagnosis necessary? Am J Clin Nutr 2006; 83: 1237- 47.

15. Reaven G. The Metabolic Syndrome: Requiescat in Pace. Clin Chem 2005; 51: 931-8.

16. ReAven G. Insulin Resistance, Cardiovascular Disease, and the Metabolic Syndrome. How well do the emperor's clothes fit? Diabetes Care 2004; 27; 1011-2.

17. Grundy S. Metabolic Syndrome: Connecting and Reconciling Cardiovascular and Diabetes Worlds. J Am Coll Cardiol 2006; 47: 1093-100.

18. Lorenzo C, Wimams K, Hunt KJ, Haffner S. The National Cholesterol Education Program-Adult Treat- 
ment Panel III, International Diabetes Federation, and World Health Organization Definitions of the Metabolic Syndrome as Predictors of Incident Cardiovascular Disease and Diabetes. Diabetes Care 2007; 30: 8-13.

19. Bonora E, Targher G, Formentini G, Calcaterra F, LomabaRdi S, Marin F ET AL. The Metabolic Syndrome is an independent predictor of cardiovascular disease in Type 2 diabetic subjects. Prospective data from the Verona Diabetes Complications Study. Diabet Med 2004; 21: 52-8.

20. ZIcK Y. Insulin Resistance: a phosphorylation based uncoupling of insulin signaling. Trends Cell Biol 2001; 11: 437-41.

21. NeEL JV. Diabetes Mellitus: a thifty genotipe rendered cetrimental by progress? Am J Hum Genet 1962; 14: 353-62.

22. Dandona P, Aljada A, Chaudhuri A, Mohanty P, Garg R. Metabolic Syndrome: A Comprehensive Perspective Based on Interactions Between Obesity, Diabetes, and Inflammation. Circulation 2005; 111; $1448-54$

23. Goh TT, Mason TM, Gupta N, So A, Lam TK, Lam L et AL. Lipid-induced beta-cell dysfunction in vivo in models of progressive beta-cell failure. Am J Physiol Endocrinol Metab 2007; 292: E549-60.

24. WeINBERG JM. Lipotoxicity. Kidney Int 2006; 70: 15606.

25. Johnson L, Weinstock R. The Metabolic Syndrome: Concepts and Controversy. Mayo Clin Proc 2006; 8: 1615-20.

26. Guo Z, Hensrud D, Johnson M, Jensen M. Regional Postprandial Fatty Acid Metabolism in Different Obesity Phenotypes. Diabetes 1999; 48: 1586-92.

27. Appel S, Harreil J, Davenport M. Central Obesity, the Metabolic Syndrome, and Plasminogen Activator Inhibitor-1 in Young Adults. J Am Acad Nurse Pract 2005; 17: 535-41.

28. Tripathy D, Mohanty P, Dhindsa S, Syed T, Ghanim H, Aljada A, Dandona P. Elevation of free fatty acids induces inflammation and impairs vascular reactivity in healthy subjects. Diabetes 2003; 52: 2882-7.

29. Hotamisugil GS, Peraldi P, Budavari A, Eus R., White MF, SiIEGELMAN BR. IRS-1-mediated inhibition of insulin receptor tyrosine kinase activity in TNFalpha- and obesity-induced insulin resistance. Science 1996; 271: 665-8.

30. Rui L, Yuan M, Frantz D, Shoelson S, White MF. SOCS-1 and SOCS-3 block insulin signaling by ubiquitin-mediated degradation of IRS1 and IRS2. J Biol Chem 2002; 277: 42394-8.

31. Bahia L, Aguiar LG, Vilueia N, Bottino D, GodoyMatos AF, Geloneze B y cols. Relationship between adipokines, inflammation, and vascular reactivity in lean controls and obese subjects with metabolic syndrome. Clinics 2006; 61: 433-40.
32. Aljada A, Mohanty P, Ghanim $H$, Abdo T, Tripathy D, Chaudhuri $A$, Dandona $P$. Increase in intranuclear nuclear factor kappaB and decrease in inhibitor kappaB in mononuclear cells after a mixed meal: evidence for a proinflammatory effect. Am J Clin Nutr 2004; 79: 682-90.

33. Dandona P, Mohanty P, Ghanim H, Aljada A, Browne $\mathrm{R}$, Hamouda $\mathrm{W}$ ET aL. The suppressive effect of dietary restriction and weight loss in the obese on the generation of reactive oxygen species by leukocytes, lipid peroxidation, and protein carbonylation. J Clin Endocrinol Metab 2001; 86: 355-62.

34. Prasad A, Quyyum A. Renin-Angiotensin System and Angiotensin Receptor Blockers in the Metabolic Syndrome. Circulation 2004; 110: 1507-12.

35. Serne EH, de Jongh RT, ERinga EC, IJzerman RG, STEHOUwER CD. Microvascular dysfunction: causative role in the association between hypertension, insulin resistance and the metabolic syndrome? Essays Biochem 2006; 42: 163-76.

36. Albala C, Jiménez B, Perez F, Liberman C. Polimorfismo de la proteína ligante de ácidos grasos intestinal (FABP2), obesidad e insulino resistencia. Rev Méd Chile 2006; 134: 372-9.

37. Yki-JÄRvinen H. Thiazolidinediones. N Engl J Med 2004; 351: 1106-18.

38. Fourth Joint Task Force of the European Society of Cardiology and Other Societies on Cardiovascular Disease Prevention in Clinical Practice. European guidelines on cardiovascular disease preventionin clinical practice: executive summary. Eur Hearth J 2007; 28: 2375-414.

39. Rossner S, Sjostrom L, Noack R, Meinders AE, Noseda G. Weight loss, weight maintenance, and improved cardiovascular risk factors after 2 years treatment with orlistat for obesity. European Orlistat Obesity Study Group. Obes Res 2000; 8: 49-61.

40. Didangelos TP, Thanopoulou AK, Bousboulas SH. The ORListat and Cardiovascular risk profile in patients with metabolic syndrome and type 2 Diabetes (ORLCARDIA) study. Curr Med Res Opin 2004; 20: 1393-401.

41. Padwal R, Li SK, Lau DCW. Long-term pharmacotherapy for overweight and obesity. Int J Obes Relat Metab Disord 2003; 27: 1437-46.

42. James WP, Astrup A, Finer N, Hilsted J, Kopelman P, ROSSNER S. Effect of sibutramine on weight maintenance after weight loss: a randomised trial. STORM Study Group. Sibutramine Trial of Obesity Reduction and Maintenance. Lancet 2000; 356: 2119-25.

43. McNulty SJ, Ur E, Wiшams G. A randomized trial of sibutramine in the management of type 2 diabetic patients treated with metformin. Diabetes Care 2003; 26: $125-31$.

44. Despres JP, Golay A, Sjostrom L. Effects of rimonabant on metabolic risk factors in overweight patients with 
dyslipidemia. N Engl J Med 2005; 353: 2121-34.

45. Radwal R, Majumdar S. Drug treatments for obesity: orlistat, sibutramine, and rimonabant. Lancet 2007; 369: 71-7.

46. Gelfand E, Cannon C. Rimonabant: A Cannabinoid Receptor Type 1 Blocker for Management of Multiple Cardiometabolic Risk Factors. J Am Coll Cardiol 2006; 47: 1919-26.

47. Sjöström L, Lindroos A, Peltonen M, Torgerson J, Bouchard C, Carlsson B. Lifestyle, Diabetes, and Cardiovascular Risk Factors 10 Years after Bariatric Surgery. N Engl J Med 2004; 351: 2683-93.

48. Lee WJ, Huang MT, Wang W, Lin CM, Chen TC, Lai IR. Effects of obesity surgery on the metabolic syndrome. Arch Surg 2004; 139: 1088-92.

49. Deedwania PC, Hunninghake DB, Bays HE, Jones PH, CAin VA, BlasetTo JW. Effects of rosuvastatin, atorvastatin, simvastatin, and pravastatin on atherogenic dyslipidemia in patients with characteristics of the metabolic syndrome. Am J Cardiol 2005; 95: 360-6.

50. Tenenbaum A, Fisman E. Which is the best lipid-modifying strategy in metabolic syndrome and diabetes: fibrates, statins or both? Cardiovasc Diabetol 2004; 3: 10.

51. Tenenbaum A, Fisman E, Motro M, Adier Y. Atherogenic dyslipidemia in metabolic syndrome and type 2 diabetes: therapeutic options beyond statins. Cardiovasc Diabetol 2006; 5: 20.

52. Yusuf S, Gerstein H, Hooqwerf B and HOPE Study INVESTIGATORS. Ramipril and the development of diabetes. JAMA 2001; 286: 1882-5.

53. The DREAM Trial Investigators. Effect of Ramipril on the Incidence of Diabetes. N Engl J Med 2006; 355: 1551-62.
54. Lindholm, Ibsen H, Dahlof B and The LIFE Study GRouP. Cardiovascular morbidity and mortality in patients with diabetes in the Losartan Intervention For Endpoint reduction in hypertension study (LFE): a randomised trial against atenolol. Lancet 2002; 359: 1004-10.

55. Vitale C, Mercuro G, Castiglioni C, Cornoldi C, Tum A, FINI M ET AL. Metabolic effect of telmisartan and losartan in hypertensive patients with metabolic syndrome. Cardiovasc Diabetol 2005; 4: 6.

56. American Diabetes Association. Standards of Medical Care in Diabetes. Diabetes Care 2004; 27: Sup 1.

57. Kraemer de Aguiar LG, Laflor CM, Bahia L, Viliela N, WiERNSPERgER N, BotTino DA, Bouskela E. Metformin Improves Endothelial Vascular Reactivity in FirstDegree Relatives of Type 2 Diabetic Patients With Metabolic Syndrome and Normal Glucose Tolerance. Diabetes Care 2006: 29: 1083-9.

58. The UK Prospective Diabetes Study Group. Intensive blood-glucose control with sulphonylureas or insulin compared with conventional treatment and risk of complications in patients with type 2 diabetes (UKPDS 33). Lancet 1998; 352: 837-53.

59. Derosa G, D'Angelo A, Ragonest P, Ciccarem C, Piccinni MN, Pricolo F ET AL. Metabolic effects of pioglitazone and rosiglitazone in patients with diabetes and metabolic syndrome treated with metformin. Intern Med J 2007; 37: 79-86.

60. NisSEN S, Wolski K. Effect of Rosiglitazone on the Risk of Myocardial Infarction and Death from Cardiovascular Causes. N Engl J Med 2007; 356: 2457-71. 